\title{
Introduction : Ancrage et identité territoriale des systèmes agroalimentaires localisés
}

Javier Sanz Cañada et José Muchnik

\section{(2) OpenEdition}

Journals

Édition électronique

URL : http://journals.openedition.org/economierurale/2962

DOI : $10.4000 /$ economierurale.2962

ISSN : 2105-2581

Éditeur

Société Française d'Économie Rurale (SFER)

Édition imprimée

Date de publication : 21 mars 2011

Pagination : 4-10

ISSN : 0013-0559

\section{Référence électronique}

Javier Sanz Cañada et José Muchnik, «Introduction : Ancrage et identité territoriale des systèmes agroalimentaires localisés », Économie rurale [En ligne], 322 I mars-avril 2011, mis en ligne le 30 mars 2013, consulté le 01 mai 2019. URL : http://journals.openedition.org/economierurale/2962 ; DOI :

10.4000/economierurale.2962

(C) Tous droits réservés 


\title{
Ancrage et identité territoriale des systèmes agroalimentaires localisés
}

\author{
Javier SANZ CAÑADA \\ Centro de Ciencias Humanas y Sociales, CSIC \\ Économie agroalimentaire, Madrid, \\ Coordinateur du European Research Group SYAL, javier.sanz@cchs.csic.es \\ José MUCHNIK \\ INRA-SAD UMR Innovation Montpellier \\ Coordinateur du European Research Group SYAL, muchnik@supagro.inra.fr
}

\begin{abstract}
A u cours de ces dernières années, les systèmes agroalimentaires ont connu au niveau international d'importants changements du fait de la globalisation croissante des marchés. L'élargissement de l'espace économique des grandes entreprises alimentaires et de distribution a été influencé non seulement par les accords internationaux en matière de réduction des barrières douanières et non douanières, mais aussi par un changement de paradigme technologique des activités de logistique et de distribution, révolutionnées par la numérisation des technologies d'information. À ces évolutions sur le plan économique s'ajoutent les nouveaux défis sur le plan environnemental et de la sécurité alimentaire.

Dans ce contexte, la littérature spécialisée dans l'analyse spatiale des systèmes agroalimentaires porte un intérêt particulier sur la question de recherche suivante : «quels sont les facteurs qui déterminent le degré d'ancrage ou enracinement territorial des productions agroalimentaires? » L'analyse de cette interrogation permettrait de mieux comprendre d'une part l'attachement de certaines productions à un lieu donné et, d'autre part, les mécanismes à l'œuvre dans les processus de délocalisation vers d'autres lieux où les facteurs de production ont des moindres coûts. Les différentes contributions à ce numéro d'Économie rurale abordent cette question selon divers points de vue.
\end{abstract}

\section{Globalisation et territorialisation}

Les marchés de produits agroalimentaires intermédiaires et les marchés de consommation finale ont acquis des niveaux croissants de concentration, processus piloté par les grandes firmes de l'industrie agroalimentaire et de la grande distribution. Les grandes enseignes ont acquis de nouveaux avantages compétitifs par le fait de leurs grandes potentialités à s'adapter, tant au niveau organisationnel que commercial, aux multiples options d'approvisionnement mondial. Ces phénomènes de globalisation des marchés ont impulsé des processus de standardisation des productions et des marchés alimentaires, associés à d'importantes économies d'échelle et aux stratégies d'approvisionnement et de distribution des grandes entreprises. Les producteurs de commodities ont vu ainsi détériorer peu à peu leur capacité de négociation dans la chaîne alimentaire. Dans ces marchés, les stratégies compétitives price leadership prédominent.

Malgré la prédominance de ce modèle de production et de consommation, se sont développés différents types de stratégies alternatives aux processus de standardisation. L'existence de relations identitaires entre les hommes, les territoires qu'ils habitent et les aliments qu'ils consomment, joue un rôle catalyseur dans l'émergence de ces alternatives. La littérature qui aborde la dimension territoriale des productions agroalimentaires, donne une place privilégiée aux comportements des consommateurs et à son rôle dans la 
valorisation des productions locales à travers ses choix, orientés de plus en plus par la qualité et la spécificité des aliments consommés. Il s'agirait, à travers ces stratégies de différenciation, d'apporter une valeur ajoutée significative et des attributs spécifiques aux produits alimentaires, pour qu'ils accèdent à des segments et à des niches de marché caractérisés par la qualité et la typicité des produits demandés.

Nous constatons alors que les interactions entre dynamiques territoriales et dynamiques alimentaires conditionnent fortement l'évolution des sociétés rurales, la patrimonialisation de leurs ressources, la répartition entre les différents acteurs dans les chaînes de valeur, la santé des consommateurs et l'évolution de leurs cultures alimentaires. Il s'agit donc d'enjeux majeurs pour la construction de politiques agricoles et alimentaires à différentes échelles spatiales et administratives.

\section{Les systèmes agroalimentaires localisés}

Depuis un peu plus d'une dizaine d'années (Muchnik et Sautier, 1998)', les travaux de recherche autour du thème des Systèmes agroalimentaires localisés (SYAL), qui se situent à la croisée des analyses filières et des analyses territoriales, ont comme objectif d'élaborer un cadre théorique spécifique pour comprendre l'organisation et le fonctionnement d'un ensemble d'activités économiques (production, transformation, restauration...), culturelles (éducatives, festives...), récréatives (tourisme rural, formations, concours...), liées à un territoire spécifique, tant du point de vue matérielle que symbolique. Il s'agit de comprendre les synergies entre ces différentes activités territoriales pour renforcer l'ancrage des productions locales et la mise en valeur de produits alimentaires spécifiques, appelés dans la littérature produits typiques, de terroir, identitaires, ou bien, dans le cas des produits qui ont adopté des signes distinctifs, des produits issus des indications géographiques (AOP, AOC...).

L'objectif principal de ces théories est d'éveiller les fondements d'un modèle d'organisation collective à l'échelle territoriale qui, au-delà de la simple accumulation d'expériences et la génération d'économies, base son efficacité collective dans la formation de réseaux socio territoriaux liés à un certain type d'entreprenariat collectif articulé territorialement. Un élément essentiel est, à ce sujet, l'étude des phénomènes de coordination qui composent un «maillage » agroalimentaire entre acteurs économiques et sociaux, et les institutions d'un territoire déterminé (Muchnik, Sanz Cañada et Torres, 2008)². La notion de « maillage » se positionne justement à la croisée des filières et des territoires : elle renvoie à l'analyse de fonctions et de relations entre les différents acteurs et composants du tissu institutionnel local qui «font système », ainsi qu'aux nœuds où se concentrent les tensions (modalités de négociation, gestion des modèles techniques, transmission et protection de savoir-faire, contrats, mécanismes de fixation des prix...). C'est ainsi que l'analyse des fonctions, de leur rôle, de leur organisation et de leurs modalités de coordination, contribue à l'explication du fonctionnement du SYAL.

La caractérisation des SYAL s'appuie sur l'analyse de la spécificité des ressources locales qui, à travers des processus d'innovation, peuvent devenir des « actifs spécifiques »,

1. Muchnik J. et Sautier D. (1998). Systèmes agroalimentaires localisés et construction de territoires. Paris, ATP CIRAD, $46 \mathrm{p}$.

2. Muchnik J., Sanz Cañada J. et Torres Salcido G. (2008). Systèmes agroalimentaires localisés : état de recherches et perspectives. Cahiers d'Études et des Recherches Francophones /Agricultures, 17 (6), p. 513-519. 
dans le sens donné par les courants néo-institutionnalistes en sciences sociales (Williamson, 1975) ${ }^{3}$. Le concept de SYAL est alors indissociable de la diversité de réponses pour la mise en valeur des spécificités territoriales, qui est développée par les différentes formes d'organisation des relations entre homme/produit/territoire.

Les travaux sur l'approche SYAL qui concernent la mise en valeur des produits identitaires mettent en évidence deux grandes étapes articulées : la première se réfère à l'identification et à la spécification des produits, tandis que la seconde s'intéresse à l'activation des ressources locales. Les articles inclus dans ce numéro se situent sur la première de ces deux étapes, c'est-à-dire, l'analyse des processus d'identification des produits et des facteurs d'ancrage des SYAL. D'une manière complémentaire, une deuxième étape vise à rechercher les coordinations entre acteurs et les effets spatiaux des SYAL dans la gouvernance territoriale et dans le développement rural (Barjolle et al., 2007 ; Perrier-Cornet et Sylvander, 2000 ; Rallet, 2002 ; Sanz Cañada et Macías Vázquez, 2005 et 2008 ; Tregear et al., 2007) ${ }^{4}$.

Cependant, il est utile de préciser que l'objectif visé par les articles retenus prend aussi en compte, comme condition, que les facteurs d'ancrage des productions alimentaires puissent être mobilisés comme ressources, tant pour l'apport de rentes de différenciation à niveau local que pour le maintien et la mise en valeur du patrimoine territorial. En conséquence, les éléments qui constituent le patrimoine alimentaire local, sous forme de savoirs, savoir-faire, réseaux sociaux, traditions et cultures alimentaires, variétés végétales, races animales, paysages agricoles ou écosystèmes microbiens, entre autres, ne peuvent pas être considérés seulement comme des facteurs générateurs d'attributs différentiateurs des produits alimentaires, mais aussi comme des actifs spécifiques qui contribuent à la création de capital territorial.

\section{L'ancrage territorial Une conséquence des processus historiques}

Actuellement, nous sommes en présence d'un phénomène relativement paradoxale : tandis que certains produits standardisés connaissent un niveau de distribution à échelle mondiale jamais vu auparavant, les consommateurs recherchent de plus en plus leurs références identitaires à travers leur alimentation. Dans ce contexte, il émerge avec force un débat sur les processus de localisation/délocalisation des productions agroalimentaires, dans lesquels les spécificités territoriales des SYAL ont un rôle central. Au cours des dernières années, l'analyse du comportement spatial des activités agroalimentaires a été

\footnotetext{
3. Williamson O.-E. (1975). Markets and hierarchies: analysis and antitrust implications. New York, The Free Press.

4. Barjolle D., Réviron S. et Sylvander B. (2007). Création et distribution de valeur économique dans les filières de fromages AOP. Économies et Sociétés, série Systèmes Agroalimentaires, $\mathrm{n}^{\circ} 29,9 / 2007$, p. 15071524. - Perrier-Cornet P. et Sylvander B. (2000). Firmes, coordinations et territorialité. Une lecture économique de la diversité des filières d'appellation d'origine. Économie Rurale, $\mathrm{n}^{\circ} 258$, p. 79-89. - Rallet A. (2002). L'économie des proximités. Propos d'étape. Études et Recherches sur les Systèmes Agraires et Développement, $\mathrm{n}^{\circ}$ 33, p. 11-25. - Sanz Cañada J. et Macías Vázquez A. (2005). Quality certification, institutions and innovation in local agro-food systems: protected designations of origin of olive oil in Spain. Journal of Rural Studies, ${ }^{\circ}$ 21, p. 475-486. - Sanz Cañada J. et Macías Vázquez A. (2008). Appellations d'origine protégées et innovations : la filière « huile d'olive » à Sierra Mágina (Andalousie). Cahiers d'Études et des Recherches Francophones /Agricultures, 17 (6), p. 542-526. - Tregear A., Arfini F., Belletti G. et Marescotti A (2007). Regional foods and rural development: the role of food qualification. Journal of Rural Studies, $\mathrm{n}^{\circ} 23$, p. 12-22.
} 
vu notamment par l'économie régionale selon le point de vue d'analyse des facteurs de localisation, entendus comme des variables qui renforcent ou limitent les coûts et les bénéfices des entreprises dérivées de la localisation des activités agroalimentaires dans un lieu déterminé.

Dans la dernière décennie, les recherches ont commencé à s'ouvrir vers une vision plus dynamique et plus adaptée à la réalité des processus de localisation/délocalisation des activités agroalimentaires. Ainsi, un des principaux points d'intérêt est de déterminer quels sont les facteurs qui expliquent le fait que les productions agroalimentaires peuvent être délocalisées avec plus de facilité lorsque prédominent les modes de régulation sectorielle et les stratégies de price leadership que quand prévalent, au sein d'un SYAL, les modes de gouvernance territoriale et les stratégies de valeur ajoutée à travers l'articulation producteur/consommateur. Selon Zimmermann $(1998)^{5}$, le concept d'ancrage territorial répond à un «processus d'apprentissage collectif localisé dans le but de générer des ressources ». C'est grâce au fait que les processus d'ancrage territorial demandent, comme condition nécessaire au succès, que les acteurs locaux développent des stratégies collectives qui mènent à assurer leur survie économique, sociale et institutionnelle : «ce degré de pérennité des SYAL est en partie imputable aux caractéristiques du territoire dans lequel il se situe » (Frayssignes, 2001)

D'un point de vue historique, le caractère local d'une production agroalimentaire n'est pas un attribut naturel et permanent d'un territoire déterminé. Si nous regardons les produits alimentaires typiques qui existent actuellement à l'échelle mondiale, nous pouvons vérifier qu'ils se sont localisés sur leur territoire actuel à un moment donné de l'histoire et que les savoir-faire ont subi également des processus d'emprunt/ transmission/adaptation, qui ont abouti in fine à leur conférer leur localisation. Ainsi, par exemple, la tomate originaire d'Amérique latine est aujourd'hui une composante essentielle de nombreux plats traditionnels en Europe méditerranéenne où, au fil du temps, des variétés adaptées et des savoir-faire culinaires spécifiques ont été développés.

Dans ce sens, nous pouvons apprécier l'importance de donner à nos recherches une perspective historique en les situant dans la longue durée, car les liens biophysiques et sociales qui existent entre les produits et les territoires, changent selon des échelles temporelles qui dépassent largement les « temps des projets » de recherche et développement dans lesquels nous situons nos actions. Il en est de même pour l'évolution des identités alimentaires, car celles-ci constituent une référence essentielle à un moment donné mais, en même temps, elles évoluent profondément dans la durée. Il s'agit alors d'analyser historiquement les processus d'identification, c'est-à-dire les processus de construction, transformation et destruction des identités alimentaires à partir des échanges/emprunts survenus entre différentes cultures (Muchnik, 2006).

5. Zimmermann J.-B. (1998). Nomadisme et ancrage territorial : propositions méthodologiques pour l'analyse des relations firmes-territoires. Revue d'Économie Régionale et Urbaine, 98-2, p. 211-230.

6. Frayssignes J. (2001). L'ancrage territorial d'une filière fromagère d'AOC. L'exemple du système Roquefort, Économie Rurale, 264-265, p. 89-103.

7. Muchnik J. (2006) Identidad territorial y calidad de los alimentos : procesos de calificación y competencias de los consumidores. Agroalimentaria, ${ }^{\circ}$ 22, p. 89-98. 


\section{Diversité de facteurs d'ancrage territorial Les liens entre produits identitaires et territoire}

L'analyse de la typicité ou, d'un point de vue plus large, l'étude des caractéristiques des aliments locaux, devient un objectif prioritaire des recherches sur les SYAL. Cette typicité n'est pas seulement issue de facteurs naturels (climatiques, écologiques), mais « c'est un système au sein duquel s'établissent des interactions complexes entre un ensemble de facteurs humains, une production agricole et un milieu naturel » (INAO, 1992) $)^{8}$. Le terroir est le résultat d'une construction bio sociale et historique, qui suppose un processus de patrimonialisation qui associe la biodiversité agraire, les pratiques, les techniques, les savoirs, les réseaux sociaux et institutionnels, autour de la production locale d'un aliment identitaire (Deffontaines, 2005) ${ }^{9}$. Il est donc naturel que, pour mener ces recherches, nous fassions appelle à des éclairages disciplinaires différentes (agronomiques, géographiques, biologiques, économiques, anthropologiques...). Précisons encore qu'il ne s'agit pas d'une interdisciplinarité a priori ou bien d'une condition préalable, mais d'une interdisciplinarité construite dans l'action en fonction des objets de recherche traités.

Les points de vue théoriques basés sur la «biodiversité culturelle », le « savoir-faire collectif localisé », les « réseaux sociaux d'innovation et de connaissance », ou encore le concept même d'ancrage territorial, amènent à examiner, selon ces optiques distinctes et complémentaires, les liens entre les produits alimentaires et les éléments patrimoniaux qui font le territoire (Bérard et Marchenay, 2004 ; Casabianca, Béranger et Morin, 1999; Cegarra, 2005 ; Chiffoleau et Touzard, 2007 ; Frayssignes, op. cit. ; Praly et al., 2007 ; Zimmermann, op. cit. $)^{10}$. Ce type de littérature rassemble différents types de catégorisation des liens entre les produits identitaires et le territoire. Ces classifications ont comme modèle commun trois grandes catégories de liens :

(i) ceux relatifs au patrimoine naturel (biophysique);

(ii) ceux qui se réfèrent au patrimoine culturel (savoirs, identités...) ;

(iii) ceux qui se réfèrent aux réseaux socio-économiques et institutionnels. Les spécificités que présentent chacune de ces trois catégories de liens expliquent que le territoire devienne une source importante de diversité des productions agroalimentaires locales.

Plus particulièrement, une première catégorie de liens est fondée par le patrimoine naturel, dont la source de variabilité provient des caractéristiques du sol, du climat, des écosystèmes..., en somme, de la biodiversité existante dans un lieu donné. Mais la biodiversité inhérente aux SYAL est la conséquence d'un processus d'évolution qui

8. Institut National des Appellations d'Origine (1992). Rapport de politique générale. Paris, INAO, p. 26. 9. Deffontaines J.-P. (2005). Le terroir, une notion polysémique. In Bérard et al. (éds.) «Biodiversité et savoirs naturalistes locaux en France ». Paris, Éds. CIRAD, IDDRI, IFB, INRA, p. $38-43$.

10. Bérard L. et Marchenay P. (2004). Les produits du terroir. Entre cultures et règlements. Paris, CNRS Editions. Casabianca F., Béranger Cl. et Morin G. (1999). La codification des liens entre le terroir et le produit dans le cas des produits carnés : analyse de situation et perspectives. In Lagrange (coord.) «Signes officiels de qualité et développement local ». Paris, Éds. SFER et Tec \& Doc, p. 91-104. - Cegarra M. (2005). La construction du « local » en France. In Bérard et al. (eds.) «Biodiversité et savoirs naturalistes locaux en France ». Paris, Eds. CIRAD, IDDRI, IFB, INRA, p. 27-33. - Chiffoleau Y. et Touzard J.-M. (2007). Réseaux d'entrepreneurs et innovation dans un cluster : une approche par les relations de conseil. Économies et Sociétés, série Systèmes Agroalimentaires, no 29, 9, p. 1485-1506. Praly C. ; Chazoule C. ; Delfosse C. ; Pluvinage J. (2007). SYAL et relocalisation des agricultures européennes : une application à l'arboriculture de la Moyenne Vallée du Rhône, Économies et Sociétés, série « Systèmes agroalimentaires », $\mathrm{AG}, \mathrm{n}^{\circ} 29$, p. 1547-1566. 
intègre des facteurs naturels avec des savoirs spécifiques, des techniques, des savoir-faire et du patrimoine culturel agroalimentaire d'un territoire, ce qui constitue une deuxième catégorie de liens. Par ailleurs, la construction bio sociale de la typicité d'un produit alimentaire ne pourra pas se matérialiser dans un territoire donné si elle n'inclut pas la création des réseaux sociaux, économiques et institutionnels spécifiques. Ces réseaux constituent un troisième type de facteurs de variabilité qui font partie du capital social et institutionnel d'un territoire, car ils permettent d'adopter de façon collective des innovations, techniques ou organisationnelles, qui auront des impacts sur la reproduction ou la destruction de la biodiversité.

La diversité de facteurs d'ancrage territorial et leur rôle sur la pérennité des SYAL constituent, comme nous l'avons signalé, la question centrale abordée dans le présent numéro. Ceci nous amène à analyser la génération des attributs de différenciation des aliments identitaires (ou la formation de leur typicité et de leur spécificité territoriale) et à traiter deux questions de recherche étroitement associées :

- Quels sont les liens entre territoire et productions agroalimentaires?

- Quelles catégories d'actifs patrimoniaux et de capital territorial déterminent la diversité des SYAL et la typicité des produits alimentaires ?

Les contributions qui sont incluses dans ce numéro ont comme objectif général de montrer une analyse critique des facteurs d'ancrage territorial dans l'évolution des SYAL et de leur rôle dans les processus de localisation / délocalisation des productions agroalimentaires locales.

Les trois premiers travaux font référence, à travers différents points de vue théoriques, aux SYAL qui présentent une certaine profondeur historique, avec différents types de liens au territoire (biophysiques, culturaux, socioéconomiques).

- L'article de Carole Chazoule et Rémy Lambert élabore une caractérisation de la diversité des différents types de liens au territoire existants dans le domaine des productions typiques du Québec, qui appartiennent à différents secteurs alimentaires. Les uns jouissent d'une reconnaissance de leur typicité par les consommateurs et certains sont en voie d'obtenir une indication géographique, alors que d'autres produits ne bénéficient pas de cette reconnaissance. Les auteurs soulignent l'influence de l'ancrage territorial sur les potentialités de mise en valeur des aliments, dans un contexte géographique où prédominent largement les productions standardisées.

- Les articles de Pascale Moity Maïzi et de Rémi Bouche ainsi que celui d'Adeline Lambert-Derkimba, Etienne Verrier et François Casabianca abordent le processus de construction patrimoniale et les facteurs d'ancrage de deux productions typiques qui n'ont pas atteint la reconnaissance d'une appellation d'origine protégée (AOP).

Le travail de Pascale Moity Maïzi et de Rémi Bouche sur les fromages typiques corses, introduit le concept de « savoir faire collectif ancré territorialement », qui mobilise des outils théoriques de la technologie culturelle et de l'ergonomie cognitive pour s'interroger sur les mécanismes à travers lesquels le savoir-faire pastoral et le savoir-faire fromager pourraient s'articuler territorialement pour obtenir une AOP. Actuellement, ces types de savoir-faire répondent à des schémas cognitifs différents et ne réussissent pas à se coordonner territorialement, ce qui génère une certaine fragilité de leur ancrage territoriale.

Le texte d'Adeline Lambert-Derkimba, Étienne Verrier et François Casabianca se focalise sur le rôle du patrimoine génétique des races locales porcines comme facteur 
d'ancrage et comme actif spécifique pour la mise en valeur de la charcuterie corse, qui est actuellement en phase de demande d'une AOP. Il se détache l'importance des rôles des réseaux socio institutionnels et de l'action collective dans la définition du cahier des charges, ce qui devrait permettre de résoudre le conflit qui existe actuellement entre les contraintes à court terme et un processus de patrimonialisation à long terme qui met en valeur la spécificité génétique des races porcines locales.

De la même façon, s'il est démontré que le degré d'ancrage d'un produit à son territoire n'est pas invariable avec le temps, nous pouvons également affirmer que l'existence même d'un SYAL n'est pas garantie dans la durée. Ainsi, le quatrième et le cinquième article reflètent des situations relativement opposées.

- L'exercice de Paul Minvielle, Jean-Noël Consales et Jacques Daligaux, traitant des «Associations de Maintien de l'Agriculture Paysanne » (AMAP) en Provence, est d'analyser l'émergence d'un nouveau SYAL basé sur une agriculture périurbaine consacrée à la production de fruits et au maraîchage, avec certification biologique. L'AMAP est arrivée à bénéficier d'un solide ancrage territorial dans un laps de temps relativement court. Ce type de SYAL se caractérise par l'organisation en aval des associations de consommateurs qui impulsent de nouvelles conventions et de relations contractuelles avec les agriculteurs périurbains en circuits courts. Dans ce cas, les caractéristiques socioculturelles des consommateurs urbains sont un facteur d'ancrage très influent, tant sur la spécificité que sur la gouvernance territoriales du SYAL.

- Au contraire, l'article de Julio Elverdin, Andrea Maggio et José Muchnik étudie l'évolution d'une production traditionnellement ancrée dans le territoire, comme les systèmes extensifs de viande bovine de la Pampa en Argentine, destinée à $80 \%$ au marché interne. Cette production se délocalise progressivement, à cause principalement de l'expansion de la culture de soja transgénique pour le marché extérieur. Les fortes oscillations conjoncturelles des prix des matières premières et les hauts risques de délocalisation que peut avoir la production de soja, avec un très faible ancrage territorial, sont des aspects soumis au débat dans une optique de durabilité, d'équilibre des sociétés rurales et de l'évolution des systèmes de production agricoles. Les auteurs mettent à l'épreuve la pertinence de l'approche SYAL pour analyser des systèmes au sein desquels, paradoxalement, la tendance prédominante n'est pas à l'ancrage mais à la délocalisation.

Pour conclure, les politiques d'appui au secteur agroalimentaire ont été historiquement structurées presque exclusivement par filière, ce qui est cohérent avec l'organisation professionnelle du monde agricole et agroalimentaire. Dans le contexte actuel, on constate l'émergence de nouveaux acteurs plus soucieux de l'inscription territoriale et de l'impact environnemental de leurs activités.

Les SYAL peuvent alors offrir une double contribution : sur le plan théorique, un cadre d'analyse pertinent pour comprendre l'évolution des processus de localisation/délocalisation des activités productives ; sur le plan opérationnel, un cadre d'orientation pour la restructuration de politiques publiques et pour l'organisation de projets de développement territorial qui visent une juste articulation entre compétitivité économique, dynamiques sociales et contraintes environnementales. 